\title{
Study of Raman Spectroscopy to detect the Underlying Substance Concealed below Diffusely Scattering Medium
}

\author{
Xiaohua Zhang*, Ji Zhang, Haifeng Zhang, Jianxin Lu, Leijian Wang, Yongsheng Xu \\ China Institute of Atomic Energy, Beijing, China \\ Email: *xhciae@126.com
}

Received 2013

\begin{abstract}
Measurement and comparison of $\mathrm{NaNO}_{3}$ powder concealed in opaque and semi-transparent plastic bottles are carried out through conventional Raman spectroscopy and spatially offset Raman spectroscopy individually. The action mechanism why the spatially offset Raman spectroscopy can effectively detect the medium concealed in the nontransparent bottle is analyzed. The spatially offset Raman spectroscopy breaks through the detection neck of the conventional Raman spectroscopy (the detection depth is small and cannot detect the ingredient of the subsurface under non-transparent medium), and the measurement and identification of the substance concealed in the non-transparent medium (opaque/semi-transparent plastic) bottle have been realized.
\end{abstract}

Keywords: Spatially Offset Raman Spectroscopy; Opaque/sub-transparent Plastic Bottle; Ingredient of the Subsurface; Diffuse Scattering

\section{Introduction}

Raman spectroscopy is a measurement method based on the inelastic scattering through interaction between light and matter. It can test samples noncontactly and nondestructively, and offers a substantially higher degree of chemical specificity, so this technique is one of the research focuses of analytical science. To conventional Raman spectroscopy adopted generally, the sampling depth is only limited in the area no more than several hundred microns of depth [1], or subsurface in transparent medium. However, diffusely scattering surface layers are frequently encountered in many medical and biological applications, such as food products, colloids, polymers, catalysts, powders in general, coating technology, dermatology applications involving the detection of cancerous tissue, biochemical identification for medical studies, and noninvasive authentication of pharmaceutical products. Such samples are often highly heterogeneous and can be made of various layers, each having a different chemical make-up. The major goal for analytical science is to provide a method capable of determining the chemical composition of subsurface layers in turbid media in a non-destructive way, by which the sample can be authenticated or the disease can be diagnosed. If the conventional Raman spectroscopy is employed, the fluorescence and Raman spectra of the target are collected simultaneously, whereas the Raman spectrum of the sub-

${ }^{*}$ Corresponding author. surface is relatively very weak, and it is often interfered seriously or even overwhelmed by the fluorescence and Raman spectra from the surface layer, so it becomes extremely complicated or even impossible to separate the spectrum of the surface and the one of the subsurface. In the face of these emerging challenges, a new technique based on Raman scattering, spatially offset Raman spectroscopy (SORS), which is capable of retrieving the subsurface concealed by opaque or sub- transparent diffusely scattering medium, is discovered by the workers of Rutherford Appleton laboratory [2]. And it has been used in the fields such as researches and applications of authentication of the packed pharmaceutical products [3-5], disease diagnoses [6-8], anti-smuggling [9], airport screening for explosives especial liquid ones [10-12], and has gained extraordinary attention and approval from the whole society.

In this paper, measurement and contrasting analysis for $\mathrm{NaNO}_{3}$ powder concealed in opaque and sub-transparent bottles have been carried out through conventional Raman configuration and SORS configuration individually. And the action mechanism how the SORS can realize the efficient detection of the media concealed in nontransparent plastic bottles has been analyzed.

\section{Thoery and Method}

During Raman scattering, the incoming light passing through the sample will be inelastically scattered from the molecular vibration, which causes the energy (wave- 
length/ frequency) of the photons to be altered. In the conventional mode, backscattering geometry is often adopted whose focus of the collecting lens is strictly overlapped with the illuminated spot. However, the SORS is based on the collection of the Raman spectrum emanating from spatially offset regions away from the point of illumination $\Delta \mathrm{s}$ on the sample surface, see Figure 1, where the relative intensity ratio of Raman spectrum of the surface to the one of the subsurface varies with the different offset distance. If the spectra data of spatial offset distance $\Delta \mathrm{s} \neq 0$ and $\Delta \mathrm{s}=0$ are processed, the pure Raman spectrum of the subsurface covered by the opaque/sub-transparent medium will be retrieved.

\section{Experiment}

\subsection{Experiment Apparatus}

The setup diagram of the SORS of the experiment is shown in Figure 2, the probe beam is generated by a temperature stabilized $532 \mathrm{~nm}$ constant wave laser (MGL-III -532 , Changchun New Industries), the output power of which is $150 \mathrm{~mW}$ with spectral line width is $<0.2 \mathrm{~nm}$. The illuminating laser light is incident on the sample at $\sim 45^{\circ}$ after it is focused by lens $\mathrm{F}_{1}$. Here, mirror $\mathrm{M}$ and the sample are all placed on a 1D micro-positioning stage, keeping the point of incidence on the sample fixed with respect to the sample. In the configuration, a lens $\mathrm{F}_{2}$ with $20 \mathrm{~mm}$ diameter and $50 \mathrm{~mm}$ focal length is adopted for spectra collecting and collimating, then the interaction zone of laser and the sample is imaged onto the fiber detector (a single fiber with $1 \mathrm{~mm}$ diameter) with the ratio of $1: 1$ by $F_{3}$ with specifications as same as $F_{2}$. The holographic notch filter centered at $532 \mathrm{~nm}$ (Kaiser Optical Systems, Inc.) between $F_{2}$ and $F_{3}$ is the crucial element, which can reduce the intensity of Rayleigh scattering by three orders of magnitude, whereas the Raman scattering light is of no attenuation. The spectra collected are measured by the miniature spectrometer (BTC112E series) produced by $\mathrm{B} \& \mathrm{~W}$ Tek Inc.

If the mirror $\mathrm{S}$ in Figure $\mathbf{2}$ is removed, then the setup will be the conventional one, where the illuminating laser is coupled into the Raman collecting system directly and normally incident on the sample as Figrue 1(a).

\subsection{Samples and Measurements}

The samples used in the experiments are chemical reagent $\mathrm{NaNO}_{3}$ produced by Beijing Red Star Chemical Factory, white plastic bottle(opaque) with thickness of $1 \mathrm{~mm}$ and Olay oil moisture protection cream bottle (subtransparent hard plastic, thickness of about $6 \mathrm{~mm}$ ), and $\mathrm{NaNO}_{3}$ packed in the bottles aforementioned. The chemical reagent $\mathrm{NaNO}_{3}$ is fine powder ground by a pestle and mortal, which is loosely packed in the bottles or piled on a metal plate without any mechanical tapping.
Raman spectra of powder $\mathrm{NaNO}_{3}$, opaque and subtransparent plastic bottles, and bottles containing $\mathrm{NaNO}_{3}$ are measured by the conventional setup, and the spatially offset ones with different offset distance of bottles containing powder $\mathrm{NaNO}_{3}$ are then measured by the SORS configuration in Figure 2.

\section{Results and Discussion}

Firstly, Raman spectra of powder $\mathrm{NaNO}_{3}$, opaque plastic bottle with thickness of about $1 \mathrm{~mm}$, and the bottle containing $\mathrm{NaNO}_{3}$ powder are individually measured by the conventional setup, and the results are shown in Figure 3.

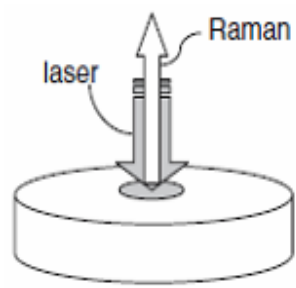

(a)

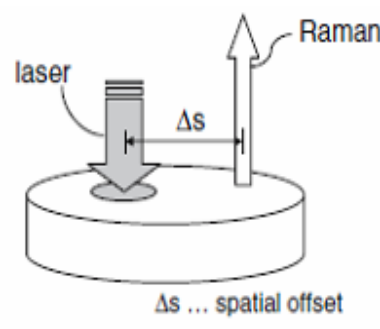

(b)
Figure 1. Schematic diagram for (a) backscattering Raman spectroscopy and (b) spatially offset Raman spectroscopy.

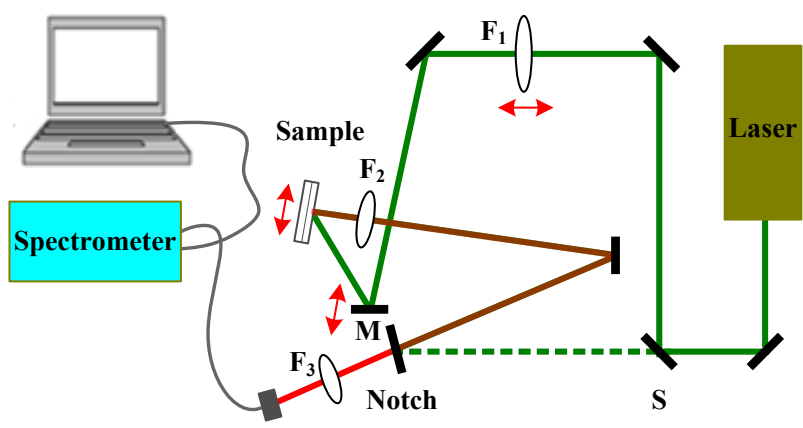

Figure 2. Setup of spatially offset Raman spectroscopy.

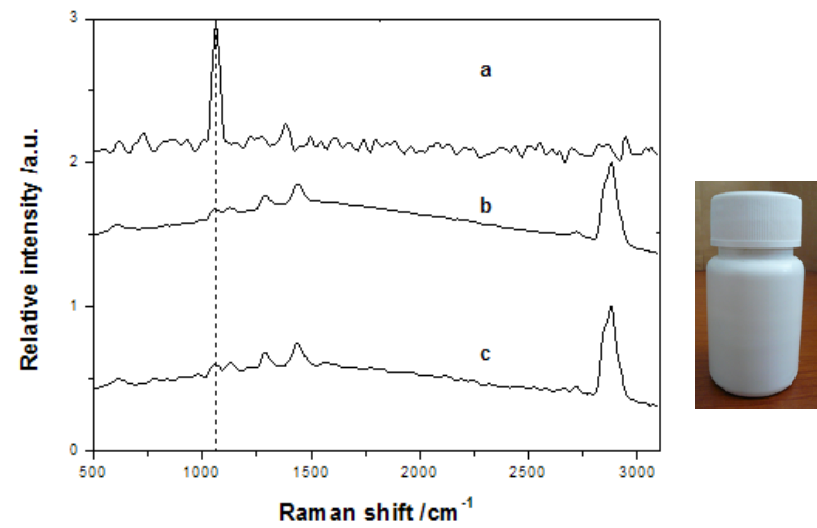

Figure 3. Conventional Raman spectroscopy of $\mathrm{NaNO}_{3}$ powder, white plastic bottle (thickness of $\sim 1 \mathbf{~ m m}$ ), and the bottle containing $\mathrm{NaNO}_{3}$ : (a) $\mathrm{NaNO}_{3}$; (b) bottle containing $\mathrm{NaNO}_{3}$; (c) empty bottle. 
As shown in the figure, there is a sharp Raman peak of the $\mathrm{NO}_{3}{ }^{-}$in the spectrum of $\mathrm{NaNO}_{3}$ piled on the metal plate, however, the Raman spectrum of $\mathrm{NaNO}_{3}$ concealed in the plastic bottle is remarkably similar to the one of the empty bottle, without obviously peak near $1062 \mathrm{~cm}^{-1}$ at all. All these results indicate that it is very efficient for the conventional Raman spectroscopy to probe the near surface ingredient, nevertheless, this approach is hampered or fails when facing to the detection of the subsurface concealed in the opaque plastic bottle, because the illumination and collection zones are coincided, the fluorescence and Raman spectra generated from the bottle are intense enough to 'swamp' the detector, thus degrading or precluding entirely, the detection of the relatively much weaker Raman photons from the deep layer.

Spectra shown in Figure 4 are the Raman spectra of the $\mathrm{NaNO}_{3}$ powder in Figure 3, where the Raman spectrum collection zones are offset from the illumination zone with different distances, here the Raman peak intensity of the plastic bottle is normalized. Seen from above figure, the ratio of the relative Raman intensities of $\mathrm{NO}_{3}{ }^{-}$to the plastic bottle increases with the spatial offset distance, that is, SORS depresses the interference of the Raman and fluorescence coming from the surface layer. However, when the offset distance is more than $6 \mathrm{~mm}$, the ratio of the signal to the noise becomes worse, and the increase of the relative intensity of $\mathrm{NO}_{3}{ }^{-}$Raman spectrum becomes unobvious. Nevertheless, the penetration depth of the SORS to the opaque medium is far more than the conventional Raman spectroscopy, and the detection and identification of the subsurface in the opaque plastic bottle can be realized.

To the detection of the subsurface concealed in the sub-transparent medium, Raman spectra of powder $\mathrm{NaNO}_{3}$, $\mathrm{OLAY}^{\circledR}$ moisture protection cream bottle (thickness of about $6 \mathrm{~mm}$, sub-transparent) containing $\mathrm{NaNO}_{3}$ and the empty bottle measured by the conventional Raman spectrum configuration are shown in Figrue 5. As same as the spectra in Figure 3, the Raman spectrum of the bottled $\mathrm{NaNO}_{3}$ is extremely similar with the one of the empty bottle, without obvious $\mathrm{NO}_{3}{ }^{-}$Raman peak near $1062 \mathrm{~cm}^{-1}$ at all. And compared with spectrum of the empty bottle, there is only a little difference near $1062 \mathrm{~cm}^{-1}$. Therefore, it is difficult to authenticate the ingredient in the subtransparent bottle according to the spectral data in Figure 5.

The SORS of $\mathrm{NaNO}_{3}$ contained in the sub-transparent plastic bottle with different spatially offset distance are shown in Figure 6, where Raman peak intensity of the bottle is normalized. Compared with the conventional one, the relative intensity of $\mathrm{NO}_{3}{ }^{-}$near $1062 \mathrm{~cm}^{-1}$ in the SORS increased with the spatial offset distance, and the clear Raman peak is already present at $\Delta \mathrm{s}=3 \mathrm{~mm}$, that is, the interference of the Raman and fluorescence signals from the surface has been depressed to a great extent.
Seen from above experimental study, the conventional Raman spectrum emphasizes the signals emitted from the container wall (although a contribution from the concealed substances may also be apparent). The SORS is also taken at the surface of the container, but at a position spatially offset from the illumination zone, and this measurement is effective at capturing subsurface photons, owing to the ability to achieve significant suppression of the surface Raman and fluorescence signals, thereby the efficient detection of the substance concealed in nontransparent diffusely scattering bottles can be carried out. The action principle of the latter is as follows_(Figure 7): When the illuminating laser incident on the sample penetrates (with exponentially intensity) into the diffusely scattering sample, the original straight trajectory of the photons is rapidly converted to a range of sideways scatters, which eventually completely randomize the path of the photons after being scattered several times. Provided the bottle wall is not too thick, some of the photons will

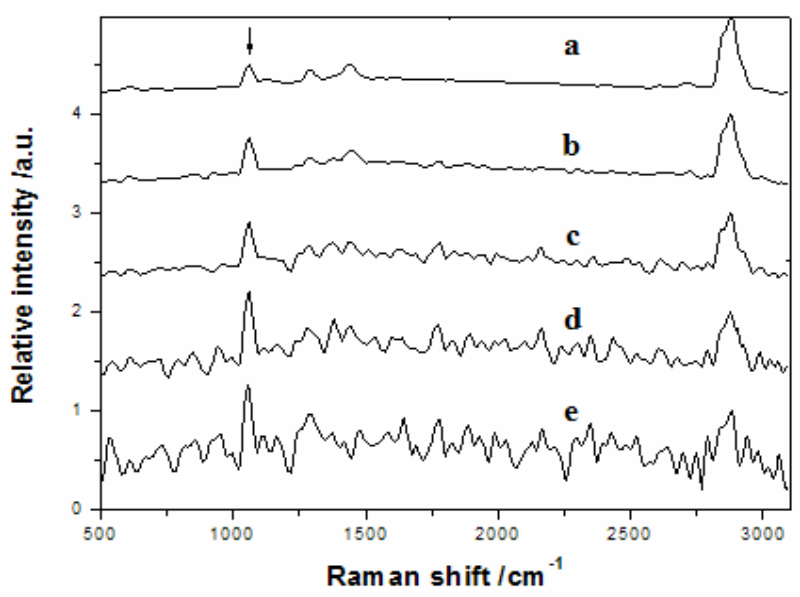

Figure 4. SORS with different spatial offset of $\mathrm{NaNO}_{3}$ contained in white plastic bottle (Raman peak intensity of the bottle is normalized): (a) $1.5 \mathrm{~mm}$ offset; (b) $3.0 \mathrm{~mm}$ offset; (c) $4.5 \mathrm{~mm}$ offset; (d) $6.0 \mathrm{~mm}$ offset; (e) $7.5 \mathrm{~mm}$ offset.

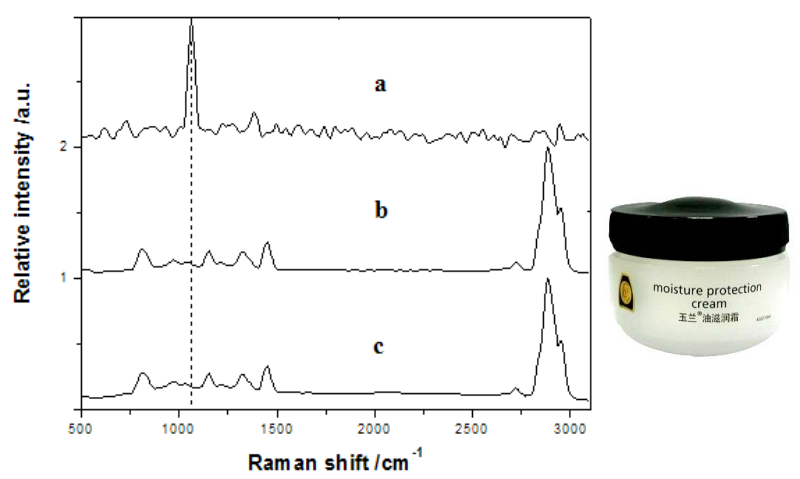

Figure 5. Conventional Raman spectroscopy of $\mathrm{NaNO}_{3}$, OLAY $^{\circledR}$ moisture protection cream bottle containing $\mathrm{NaNO}_{3}$ and the empty bottle: (a) $\mathrm{NaNO}_{3}$; (b) bottle(thickness of 6mm) containing $\mathrm{NaNO}_{3}$; (c) empty bottle. 


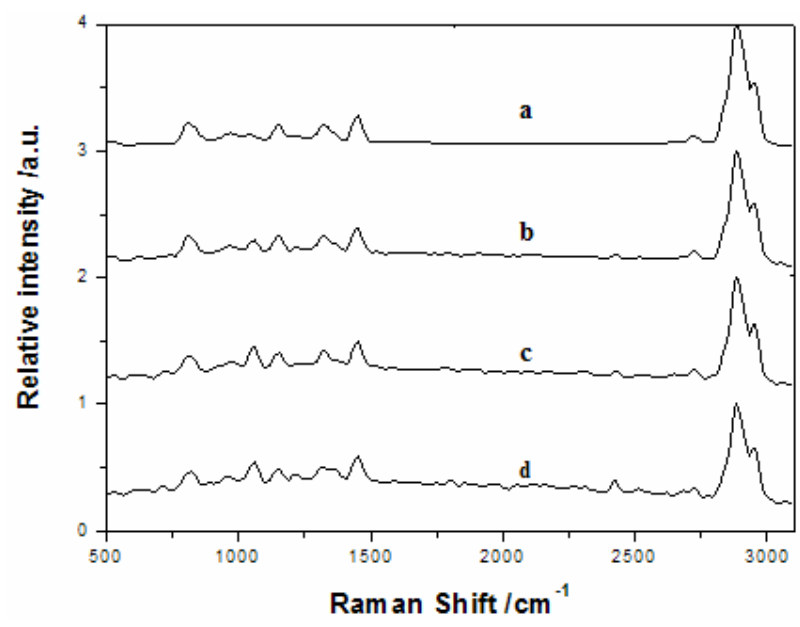

Figure 6. Contrast between SORS with different spatially offset and conventional Raman spectrum of OLAY ${ }^{\circledR}$ moisture protection cream bottle containing $\mathrm{NaNO}_{3}$ (Raman peak intensity of the bottle is normalized): (a) conventional Raman spectrum; (b) $1 \mathrm{~mm}$ offset; (c) $3 \mathrm{~mm}$ offset; (d) 5 mm offset.

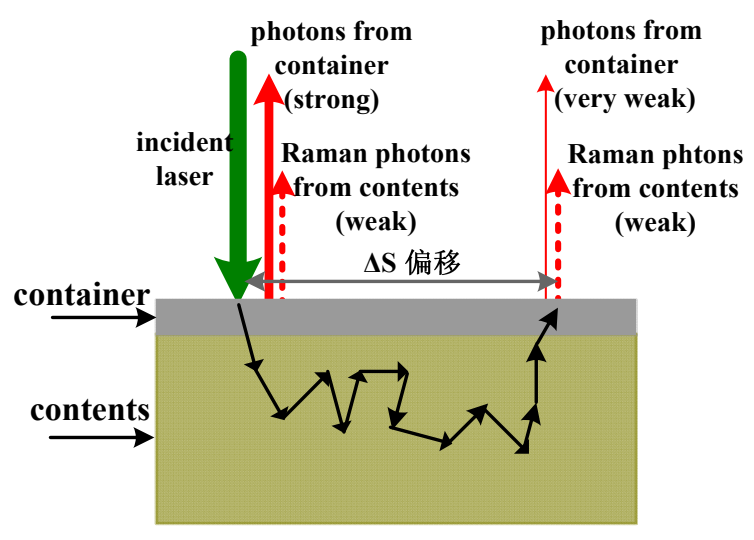

Figure 7. Diagram of the SORS action principle.

reach the $\mathrm{NaNO}_{3}$ in the bottle, whereafter they will be randomized by $\mathrm{NaNO}_{3}$ powder. And those photons that are randomly scattered back toward the bottle wall will reemerge. On average, the deeper the photon has travelled into the bottle, the further away from the original point of entry that it reemerges from the bottle surface[2]. Most of the photons collected from the point offset from the entry point $\Delta \mathrm{s}$ are Rayleigh scattered ones. Nevertheless, some of them will involve Raman scattering ones taking information of the sample. Collecting these photons originating at an appropriate offset will thereby bias the collection towards the Raman signals of the underlying $\mathrm{NaNO}_{3}$. In comparison, the Raman photons originating from the plastic bottle decay very rapidly with increasing offset from the illuminating point, since they have had much less opportunity to travel sideways by scattering, than those emerging from the underlying layers[2]. Consequently, SORS technique has the ability to depress the Raman and fluorescence signals emanating from the surface (bottle wall) significantly, breaking through the neck of the Raman spectroscopy detection and realizing the probing of the substance concealed in the diffusely medium (opaque or sub-transparent plastic bottle). Therefore, SORS has advantages that the conventional Raman spectroscopy can't provide at the detection of the underlying substance below diffusely scatering nontransparent medium.

\section{Conclusions}

Raman spectra of $\mathrm{NaNO}_{3}$ powder concealed in nontransparent media (opaque and semi-transparent plastic bottles) are studied using conventional Raman spectroscopy and the SORS individually. The comparison between the two kinds of technique for detection shows that the conventional one only adapts to the probe of near surface ingredient of the nontransparent media, whereas SORS has further penetration depth than the former, and it can depress the fluorescence and Raman spectrum of the surface (plastic bottles) to a great extent, by which the probe for the subsurface concealed in the nontransparent medium (opaque and sub-transparent plastic bottles) can be realized. The further work is in progress.

\section{REFERENCES}

[1] Colin J. H. Brenan and Ian W. Hunter. "Volumetric Raman microscopy through a turbid medium," Journal of Raman Spectroscopy, Vol. 27, 1996, pp. 561-570. doi:10.1002/(SICI)1097-4555(199608)27:8<561::A ID-JRS7>3.0.CO;2-9

[2] P. Matousek, I. P. Clark, E. R. C. Draper, M. D. Morris, A. E. Goodship and N. Everall, "Subsurface probing in diffusely scattering media using spatially offset Raman spectroscopy," Applied Spectroscopy, Vol. 59, 2005, pp. 393-400. doi:10.1366/0003702053641450

[3] W. J. Olds, E. Jaatinen, P.M. Fredericks, B. Cletus, H. Panayiotou and E. L. Izake, "Spatially Offset Raman Spectroscopy(SORS) for the Analysis and Detection of Packaged Pharmaceuticals and Concealed Drugs," Forensic Science International, Vol. 212, 2011, pp. 69-77. doi:10.1016/j.forsciint.2011.05.016

[4] C. Ricci, C. Eliasson, N. A. Macleod, P. N. Newton, P. Matousek and S. G. Kazarian, "Characterization of Genuine and Fake Artesunate Anti-Malarial Tablets Using Fourier Transform Infrared Imaging and Spatially Offset Raman Spectroscopy through Blister Packs," Analytical Bioanalytical Chemistry, Vol. 389, 2007, pp. 1525-1532. doi:10.1007/s00216-007-1543-1

[5] C. Eliasson and P. Matousek, "Noninvasive Authentication of Pharmaceutical Products through Packaging Using Spatially Offset Raman Spectroscopy," Analytical Chemistry, Vol. 79, 2007, pp. 1696-1701.

doi:10.1021/ac062223z 
[6] N. Stone, R. Baker, K. Rogers, A. W. Parker and P. Matousek. "Subsurface Probing of Calcifications with Spatially Offset Raman Spectroscopy (SORS): Future Possibilities for the Diagnosis of Breast Cancer," Analyst, Vol. 132, 2007, pp. 899-905. doi:10.1039/b705029a

[7] P. Matousek, "Deep Non-Invasive Raman Spectroscopy of Living Tissue and Powders," Chemical Society Reviews, Vol. 36, 2007, pp. 1292-1304. doi:10.1039/b614777c

[8] P. Matousek, E.R.C.Draper, A. E. Goodship, I. P. Clark, K. L. Ronayne and A. W. Parker, "Noninvasive Raman Spectroscopy of Human Tissue in Vivo," Applied Spectroscopy, Vol. 60, 2006, pp. 758-763. doi:10.1366/000370206777886955

[9] M. D. Hargreaves, N. A. Macleods and P. Matousek, "Application of Portable Raman Spectroscopy and Benchtop Spatially Offset Raman Spectroscopy to Inter- rogate Concealed Biomaterials," Journal of Raman Spectroscopy, Vol. 40, 2009, pp. 1875-1880.

doi:10.1002/jrs.2335

[10] B. J. Cletus, W. Olds, E. L. Izake, S. Sundarajoo, P. M. Fredericks and E. Jaatinen, "Field Portable Time Resolved SORS Sensor for the Identification of Concealed Hazards," Proceedings of SPIE, Vol. 8374, 2012, p. 837403. doi:10.1117/12.918981

[11] M. D. Hargreaves and P. Matousek, "Threat Detection of Liquid Explosive Precursor Mixtures by Spatially Offset Raman Spectroscopy(SORS)," Proceedings Of SPIE, Vol. 7486, 2009, p. 74860B. doi:10.1117/12.840195

[12] M. Bloomfield, P. W. Loeffen and P. Matousek, "Detection of Concealed Substances in Sealed Opaque Plastic and Colored Glass Containers Using SORS," Proceedings Of SPIE, Vol. 7838, 2010, p. 783808.

doi:10.1117/12.874724 